\title{
ARTICLE Chronic maternal interleukin-17 and autism-related cortical gene expression, neurobiology, and behavior
}

Serena Banu Gumusoglu ${ }^{1,2,3}$, Benjamin Wen Qing Hing ${ }^{2,3,4}$, Akanksha Sri Satya Chilukuri ${ }^{2,3}$, Jessica Jolynn Dewitt ${ }^{2,3}$, Sabrina Marie Scroggins ${ }^{5}$ and Hanna Elizabeth Stevens (iD) ${ }^{1,2,3}$

Chronic inflammation during pregnancy (e.g., preeclampsia, diabetes) is linked to increased risk for offspring neurodevelopmental disorders such as autism spectrum disorder (ASD). However, mediators of such exposures that could be targeted with maternal intervention are unclear, as few chronic gestational inflammation models have been tested. One potential mediator is interleukin17 (IL-17), a pro-inflammatory cytokine implicated in neurodevelopmental disorders and gestational disease. To test chronic maternal IL-17 impacts on offspring, C57BL/6J dams were administered IL-17A continuously throughout pregnancy. Offspring were assessed for body weight; cortical volume, gene expression, and cellular composition; and adult behavior. IL-17A-condition offspring exhibited decreased somatic and cortical size at embryonic day 18 (E18) and as adults. mRNA sequencing of E18 cortex revealed 320 differentially expressed genes in males, but none in females. These were significantly enriched for ASD (Simons Foundation Autism Research Initiative), synaptic, and cell cycle genes. By adulthood, neocortical glial cell density and gene expression were decreased, while GABAergic synaptic gene expression was increased in males. Furthermore, IL-17A-condition male but not female offspring exhibited reduced anxiety-like behavior. Social approach deficits in males were negatively correlated with neocortical GABAergic synaptic gene expression. Chronic gestational IL-17A was sufficient to cause ASD-like phenotypes early and persistently in male offspring. This echoes the male bias, altered cortical development, and behavioral findings in ASD, suggesting that chronic maternal IL-17 contributes to offspring ASD pathogenesis. Furthermore, the trajectory from embryonically dysregulated synaptic and cell cycle genes to disrupted adult glia, inhibitory synapses, and behavior suggests a mechanism for chronic maternal IL-17 effects on offspring.

Neuropsychopharmacology (2020) 45:1-10; https://doi.org/10.1038/s41386-020-0640-0

\section{INTRODUCTION}

Chronic inflammation in human pregnancy occurs in multiple disease states (e.g., preeclampsia, diabetes, psoriasis, and arthritis), and may be a pathogenic component of neurodevelopmental disorders. For example, ASD rates are significantly increased in children of preeclamptic (odds ratio: 1.85) [1] and diabetic pregnancies (hazard ratio 1.42) [2]. Gaps remain, however, in understanding how chronic gestational inflammation alters offspring brain development.

Few animal models have examined chronic gestational inflammation [3], despite clinical links with neurodevelopmental risk. Among the inflammatory maternal factors dysregulated in chronic gestational conditions like preeclampsia, interleukin-17 (IL-17), a pro-inflammatory cytokine, has been repeatedly implicated [4-6]. Links between ASD and dysregulated IL-17-secreting CD4+ T helper 17 (Th17) lymphocytes, elevated IL-17A levels [7], and IL-17 gene copy number variants [8] suggest that chronic IL-17 disruption contributes to ASD. Acute maternal immune activation (MIA) effects on ASD-relevant phenotypes in mice require maternal IL-17A and Th17 lymphocytes [9]. Together with the clinical literature, this indicates that IL-17 may be critical in chronic gestational inflammation risk for neurodevelopmental disorders.
To assess this role of chronic maternal IL-17, we exposed dams to IL-17A continuously throughout gestation. We found that chronic maternal IL-17A altered offspring embryonic cortical morphogenesis and transcriptomics, as well as adult neocortical neurobiology and behavior, specifically in males.

\section{MATERIALS AND METHODS}

Mice and infusions

C57/BI6J mice (Jackson, Bar Harbor, ME, USA) were bred, and upon detection of a vaginal plug [embryonic day (E)0], dams were singly housed to avoid minipump disturbance. Osmotic minipumps (\#1004, Alzet, Cupertino, CA, USA, $25 \mathrm{ng} / \mathrm{h}$ ) were implanted subcutaneously 3 days prior to breeding. Pumps were loaded with either IL-17A (released at $25 \mathrm{ng} / \mathrm{h}$; PeproTech, London, UK) or $0.9 \%$ sterile $\mathrm{NaCl}$ (saline; released at $0.11 \mu \mathrm{L} / \mathrm{h}$ ) and submerged in $23^{\circ} \mathrm{C}$ saline $12 \mathrm{~h}$ before implantation. This dose models serum increases with acute maternal Polyl: $\mathrm{C}$ in mice [9] and chronic gestational conditions in women (e.g., preeclampsia and diabetes) [4-6] accounting for rodent-human pharmacodynamic differences.

\footnotetext{
${ }^{1}$ Interdisciplinary Graduate Program in Neuroscience, University of lowa, lowa City, IA, USA; ${ }^{2}$ lowa Neuroscience Institute, University of lowa, lowa City, IA, USA; ${ }^{3}$ Department of Psychiatry, Carver College of Medicine, University of lowa, lowa City, IA, USA; ${ }^{4}$ Molecular Physiology and Biophysics, Carver College of Medicine, University of lowa, lowa City, IA, USA and ${ }^{5}$ Department of Obstetrics and Gynecology, Carver College of Medicine, University of lowa, lowa City, IA, USA

Correspondence: Hanna Elizabeth Stevens (hanna-stevens@uiowa.edu)
}

Received: 6 June 2019 Revised: 6 February 2020 Accepted: 11 February 2020

Published online: 19 February 2020 
Offspring was group-housed by sex. Experimental procedures involving animals (Supplementary Table 1 [10]) were approved by University of lowa Institutional Animal Care and Use Committee.

Protein analyses

Intra-litter pooled E18 placenta and fetal kidney protein were extracted with protease buffer (Roche, Indianapolis, IN, USA) and phosphatase inhibitors (Roche). Homogenate IL-17A and E18 maternal serum IL-17A and IL-6 were assessed via ELISA (Invitrogen, Carlsbad, CA, USA; four saline, three IL-17A litters and dams). Tissue IL-17A was normalized to the total protein via Pierce BCA Protein Assay (Thermo Fisher, Waltham, MA, USA). Levels below ELISA detection limits were recorded as 0 .

mRNA sequencing (mRNA-seq)

E18 dorsolateral cortical total RNA was extracted from four male and four female offspring, one from each of four saline and four IL17A litters, using the RNeasy Mini Kit (Qiagen, Hilden, Germany). Four replicates is sufficient to identify significantly differentially expressed genes with an RNA-seq approach in stress and autism models [11, 12]. In addition, differentially expressed counts detected via RNA-seq plateau with more than four replicates [13]. RNA integrity (RNA 6000 Nano kit, Agilent, Santa Clara, CA, USA) and quality (2100 Bioanalyzer Tapestation, Agilent) (RIN $\geq 8$ ) were checked. Successful mRNA-seq library preparation (TruSeq $^{\circledR}$ Stranded mRNA Library Prep, Illumina, Madison, WI, USA) was ascertained using DNA 1000 kit (Agilent) and 2100 Bioanalyzer Tapestation. Libraries were sequenced across two lanes (HiSeq 4000, Illumina) with 150-bp paired-end reactions.

Quantitative polymerase chain reaction

Dorsolateral E18 or adult neocortex (one per sex from three-four litters per condition) was processed for the total mRNA (RNeasy Mini Kit, Qiagen). The total RNA was assessed (Nanodrop Spectrophotometer, Thermofisher), and equivalent amounts reverse transcribed to complementary DNA (CDNA) (AMV First Strand cDNA Synthesis kit, BioLabs, Ipswich, MA, USA). Gene expression was assessed (SYBR Green primers; Supplementary Table 2) via qPCR in triplicate (ViiA 7 Real-Time PCR System, Thermofisher), calculated from average $\mathrm{Ct}$ values normalized to GAPDH (formula: $2^{(-\mathrm{Ct})}$ ).

\section{Immunohistochemistry}

Fixed (4\% PFA) brains from five or six males and females (one to two from three litters per condition) were sectioned (adult: $50 \mu \mathrm{m}$; E18: $25 \mu \mathrm{m}$ ) and immunostained, as described previously [14], with primary antibodies for Gaba (1:500, SigmaAldrich, St. Louis, MO, USA), S100 $\beta$ (1:500, Sigma-Aldrich), NeuN (1:300, Cell Signaling Technology, Danvers, MA, USA), Iba1 (1:500, Wako, Osaka, Japan), appropriate secondary antibodies (1:500; Molecular Probes, Eugene, OR, USA), and mounted with DAPI (Vector Laboratories, Burlingame, CA, USA). Embryonic dorsal forebrain included cortical plate, ventricular, subventricular, and intermediate zones. Adult neocortex included all six cortical layers.

\section{Stereology}

Cell populations and volumes were assessed via unbiased stereology, as described previously [15]. Briefly, Stereolnvestigator software (Microbrightfield, Colchester, VT, USA), coupled to a Zeiss Axiolmager 2 (Carl Zeiss, Oberkochen, Germany) with digital camera and motorized stage, was used to determine cell numbers and densities in pre-defined counting frames within randomly generated grids.

Previously described categories [14] were used to assess E18 lba $1^{+}$neocortical microglia.

\section{Behavior}

Adults ( $~ 8$ weeks old) from three saline and four IL-17A litters were habituated and tested during the light cycle. One assessment was performed after testing room habituation (30 min) daily. Tissues were collected at least 2 weeks after testing. Statistical outliers were removed from each task.

Three-chamber social approach. Male test mice were habituated ( $5 \mathrm{~min}$ ) to the center chamber of a three-chamber social approach apparatus (with cups in side chambers) then immediately tested for social preference with access to all three chambers $(10 \mathrm{~min})$. Forward-facing interactions between test mice and a male strainand age-matched "stranger" mouse, which had no previous interaction with the test mouse, in a mesh cup in one chamber or an empty cup in the opposite chamber (counterbalanced) were hand-coded by a blinded experimenter, who examined for the test mouse's nose passing between the cup bars. Strangers were used no more than twice daily, with at least $1 \mathrm{~h}$ between uses, and counterbalanced for their placement across groups. A ratio of "social contact" to "total contact" interactions was calculated. Locomotion was quantified using Anymaze software (Wood Dale, IL, USA).

Elevated plus maze. An elevated plus maze apparatus was used to assess mouse movement on open and closed arms (5 min). Behavior was quantified (Anymaze), and open:closed arm time ratios and percent of time spent on the open and closed arms were calculated.

Open field. Test mice were placed into the center of a rectangular, clear plexiglass arena $\left(\sim 1800 \mathrm{~cm}^{2}\right)$ for $30 \mathrm{~min}$ daily for 2 consecutive days. Daily total distance traveled, time spent in the center $75 \%$ of the arena, and intra-session habituation (difference between distance traveled during first and last $5 \mathrm{~min}$ ) were calculated (Anymaze).

Pre-pulse inhibition. Mice were placed in a tubular enclosure on an accelerometer within an isolation cabinet (SR-LAB startle response system, SD Instruments, San Diego, CA, USA) and habituated $(5 \mathrm{~min})$ to white noise $(\sim 65 \mathrm{~dB})$. In block 1 , mice underwent 10 pulse-alone trials of $120 \mathrm{~dB}$ white noise pulses for 20 ms [15 s inter-trial interval (ITI)]. In blocks 2 and 3, pre-pulses (5, 10 , or $15 \mathrm{~dB}$ for $20 \mathrm{~ms}$ ) were delivered $100 \mathrm{~ms}$ before longer pulse stimuli ( $120 \mathrm{~dB}$ for $40 \mathrm{~ms}$ ). Five trials at each dB level ( $15 \mathrm{~s}$ ITI) were randomly delivered within blocks. Startle intensity was averaged across all trials at each pre-pulse dB level. Percent animal startle inhibition at each $\mathrm{dB}$ level was calculated as follows: (pulse alone -pre-pulse)/pulse alone.

Statistical analyses

Two-way ANOVAs were used where appropriate, followed by Bonferroni's multiple comparisons tests. Corrected alpha values are reported, where appropriate. Two-sample $t$ tests or Mann-Whitney (nonparametric results) were used for other comparisons. Pearson's coefficients and two-tailed significance for behavioral and neurobiological measure correlations across individual mice were calculated. Outliers (greater than two standard deviations from the mean) were excluded. A $P$-value $<0.05$ was considered significant, and a $P$-value $<0.10$ was considered a trend.

For mRNA-Seq analyses, Illumina adaptors were removed using Trim Galore (version 0.4.1) and verified by FastQC (version 0.5.2). With Kallisto (version 0.43.1) [16-18], data were quasi-mapped to GENCODE version M16 GRCm38 (mm10) assembly and quantified using parameters -bias -bootstrap-samples $=200$-rf-stranded [16]. Average mapping efficiency ( 89\%; average 28,398,884 mapped reads per sample) was consistent with previous reports $[16,18]$. Data were pooled across lanes (average depth: 31,933,077 reads per sample) (Supplementary Table 3 ).

Differential gene expression was analyzed via high sensitivity methods (Sleuth version 0.29.0 pipeline in R statistical computing) [19] that can be validated by qPCR [20]. Briefly, transcript 
abundance estimates (Kallisto) were summarized to the gene level by normalizing transcript abundance to gene length (Sleuth). Principle component analysis was used to evaluate similarity in variance between samples. Data were analyzed with a linear model using scaled reads per base (response variable) and treatment group (explanatory variable). Sexes were analyzed separately. Batch effects were not controlled for, as library preparation was performed on all samples simultaneously and all samples were sequenced across two lanes. The Wald test was used to evaluate statistical differences between treatment groups with false discovery rate $<0.05$. Effect sizes were computed as beta values by Sleuth. Genes with $P<0.05$ were used as input for DAVID 6.8 and PANTHER 14.0 functional annotation (via functional annotation clustering and GO-Biological Process binomial overrepresentation testing, respectively) [21-23] and depicted as previously with "GOTreeVis" in R [24].

To assess mRNA-seq results for ASD candidate gene overrepresentation, differentially expressed transcripts were compared with Simons Foundation for Autism Research Initiative (SFARI) Human Genes (including 846 syndromic and category 1-4 genes downloaded May 9, 2019) using enrichment testing via one-tailed Fisher's exact test in R. This compared the number of SFARI genes identified by mRNA-seq here with the number expected by chance.

\section{RESULTS}

Gestational effects of maternal IL-17A

Continuous maternal IL-17A administration throughout gestation (Fig. 1a) increased IL-17A in maternal circulation, as expected, while IL-6, a canonical upstream IL-17 effector, was unchanged (Fig. 1b). Maternal IL-17A led to trend-increased IL-17A $[t(5)=$ 3.274, $P=0.065$ ] in E18 offspring kidney, a critical metabolic organ, but not placenta (Fig. 1C, d), and did not change litter size (Fig. 1e) or embryo resorptions (Fig. 1f). Behavioral animal litter sizes (mean litter size $=7$ saline, 6.75 IL-17A) and fecundity $(9 / 10$ in controls, $8 / 8$ in IL-17A within 5 days of mating) were unchanged. All pups were born on the morning of E19.

Maternal IL-17A throughout gestation led to lower E18 body weight, significant in males and trending in females [main effect of IL-17 treatment by two-way ANOVA $F_{1,66}=18.68, P<0.0001$; post hoc tests: males: $\downarrow 17 \% t(41)=3.995, P<0.001$, females: $\downarrow 11 \%$ $t(25)=2.379, P=0.025$; corrected alpha $=0.025$ ] (Fig. 1g). Weight decrements persisted in juveniles (main effect of IL-17 treatment by two-way ANOVA $P<0.005 ; F_{1,38}=8.948, P<0.005$ ) and adults (main effects of IL-17 treatment and sex by two-way ANOVA $P<$ $\left.0.0001 ; \quad F_{1,38}=6.473, \quad P<0.05\right)$, again significant in males only [male post hoc tests: juvenile: $\downarrow 11 \% t(24)=2.625, P=$ 0.015 ; adults: $\downarrow 7 \% t(25)=2.926, P=0.007$ corrected alpha $=0.025$; female post hoc tests: juvenile: $\downarrow 8 \% t(14)=1.904, P=0.078$; corrected alpha $=0.025]$ (Fig. 1h, i).

\section{Neurodevelopmental alterations after maternal IL-17A}

Given IL-17A-condition offspring transcriptome changes, we also investigated neurodevelopmental outcomes. Maternal IL-17A exposure reduced male offspring dorsal forebrain volume by $15 \%$ and cortical plate volume by $20 \%[t(9)=3.123, P=0.012 ; t(9)$ $=2.82, P=0.020$, respectively] (Fig. 2a). When normalized to body weight, neither volume differed between conditions, indicating symmetrical brain-body growth restriction. Cortical plate proportion of dorsal forebrain was unchanged in male offspring (Fig. 2b), reflecting similar proliferative and differentiating zone impacts. IL$17 \mathrm{~A}$ also increased dorsal forebrain total cell density in males $[t(9)$ $=2.991, P=0.015$ ] (Fig. 2c) commensurate with volume decrements, leaving total cell numbers unchanged (Fig. 2d). E18 female offspring brain outcomes were similarly changed, with 18 and $26 \%$ reductions to the dorsal forebrain and cortical plate volumes (Fig. 2e), which were approximately proportional (Fig. 2f), and increased dorsal forebrain total cell density $[t(8)=3.1, P=0.015]$
(Fig. $2 \mathrm{~g}$ ) commensurate with reduced volume such that total cell numbers remained unchanged (Fig. $2 \mathrm{~h}$ ).

Given their role as the brain's resident immune cells and ready responders to pro-inflammatory cytokines such as IL-17, microglia were assessed in E18 male offspring dorsal forebrain. Male IL-17Acondition offspring microglial number (Fig. 2i), density (Fig. 2j), or distribution across morphologies (Fig. 2k) were unchanged.

The fetal cortical transcriptome after maternal IL-17A

To examine maternal IL-17A effects on offspring neurodevelopment, we next tested for transcriptome-wide changes in E18 dorsal forebrain (neocortical precursor). mRNA sequencing (mRNA-seq) and transcriptomic analyses revealed that IL-17A exposure led to 320 differentially expressed genes in males (226 downregulated, 94 upregulated) and none in females with an FDR-corrected $(P<0.05)$ cutoff ( $n=4$ per group per sex, excluding one female IL-17A high-variance outlier) (Fig. 3a; Supplementary Table 4).

Functional annotation clustering of these 320 genes revealed significant enrichment for the "Synapse" cluster (DAVID, $P=4.0 \mathrm{E}-13$ ). Similarly, gene ontology pathway analyses identified the most significantly overrepresented biological process pathway to be "neuron-neuron synaptic transmission" (PANTHERDB, 9.11-fold enrichment, $P=1.34 \mathrm{E}-05)$. This pathway included 11 genes (Grm5, Grin2a, Grin3a, Grik4, Gabbr2, Slc17a8, Gabra1, Slc6a11, Unc13c, Gabra5, and Napb) reduced in expression in IL-17A-exposed male offspring. Other processes relevant to neurodevelopment and ASD (e.g., "neurotransmitter secretion" and "cell cycle") were also overrepresented (Fig. 3b).

Reduced expression of five and trending reduced expression of another five of the neuron-neuron synaptic transmission pathway genes was validated via $\mathrm{qPCR}$ in males. (Fig. 2c, poor Gapdh amplification excluded one IL-17A male; $n=3$ IL-17A, four saline). None of these genes were changed in females (Fig. 3d).

Due to relationships between IL-17 and ASD, we also assessed representation of differentially expressed mRNA-seq genes among those in the SFARI Human Gene Module. This revealed a highly significant overrepresentation (33 overlapping genes, $P=9.28 \mathrm{E}-4$; Supplementary Table 4, Fig. 3e), including most differentially expressed synaptic genes (Grm5, Grin2a, Grik4, Gabbr2, Gabra1, and Gabra5) and two cell cycle genes (Pax6 and Asmp).

A less stringent mRNA-seq analysis ( $P<0.05$, FDR uncorrected) revealed differentially expressed genes in males (2003) and females (677). For males, 143 were represented in the SFARI Human Gene Module data set, yielding significant overrepresentation $(P=6.47 \mathrm{E}-3)$. For females, 42 were SFARI genes, yielding no significant overrepresentation $(P=0.31)$. Gene ontology pathway analyses of differentially expressed genes revealed overrepresentation in males of similar pathways as identified with the FDRcorrected analysis (e.g., "synaptic transmission, GABAergic," 6.39fold enrichment, $P=3.31 \mathrm{E}-5$; via PANTHERDB) and "Telencephalon regionalization" in females (12.01-fold enrichment, $P=1.50 \mathrm{E}-04$; via PANTHERDB).

\section{Adult offspring brain changes after maternal IL-17A}

To determine whether growth-related alterations to IL-17A offspring cortex persisted, we assessed adult brain. As with embryonic dorsal forebrain, male neocortical volume, but not hippocampal dentate gyrus (DG) nor $C A$, was smaller after IL-17A exposure [neocortex: $t(10)=5.213, P<0.001$ ] (Fig. 4a), but not significantly different when normalized to body weight. Neocortical layer structure was unaltered in adult offspring after maternal IL-17A (Supplementary Fig. 2). Furthermore, while total neocortical cell density was unchanged, neuronal cell $\left(\mathrm{NeuN}^{+}\right)$density was significantly increased by $10 \%$ (Fig. 4b). Neurons comprised a larger proportion of neocortical cells in IL-17A male offspring (Fig. 4C), suggesting a reduced proportion of non-neuronal cells. GABAergic subtype population unchanged (average \pm SEM 


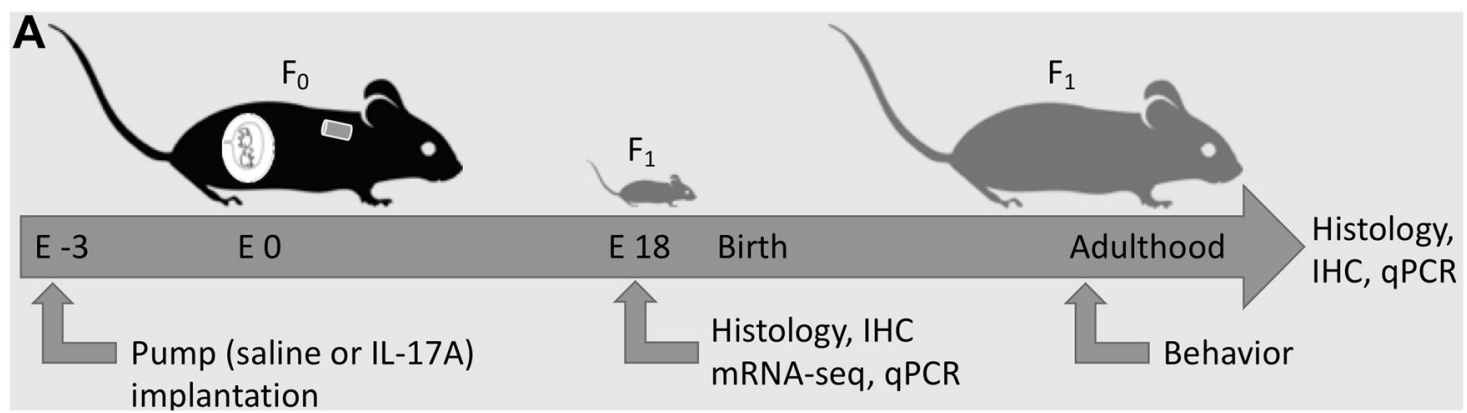

B

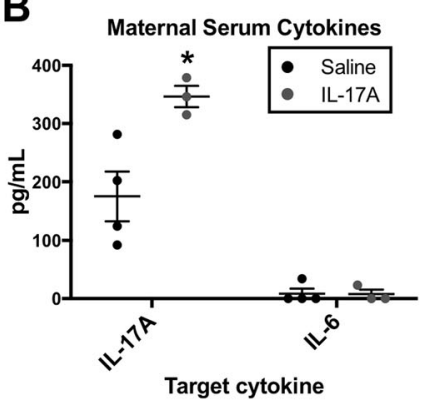

$\mathbf{G}$

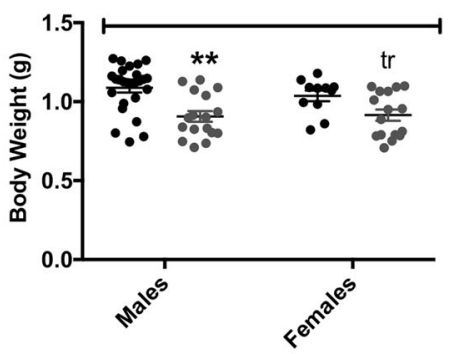

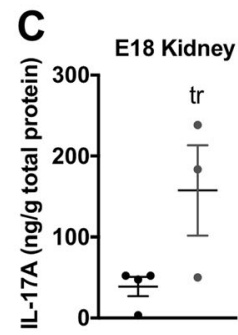

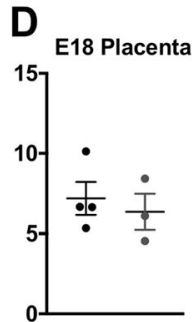

$\mathbf{E}$

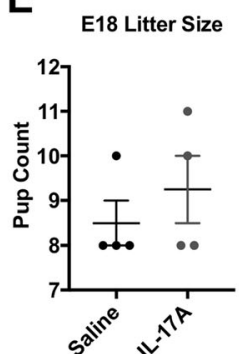

$\mathbf{F}$

E18 Resorptions

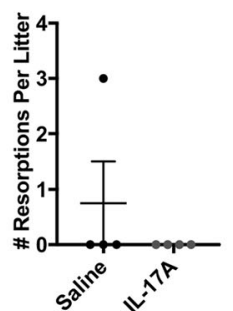

H

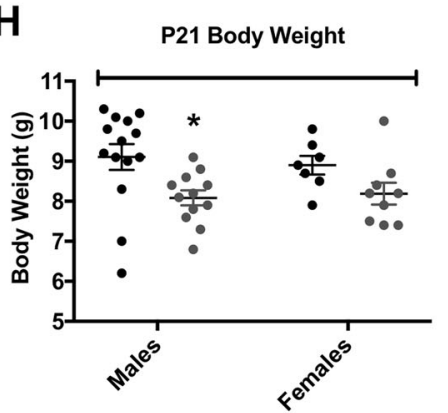

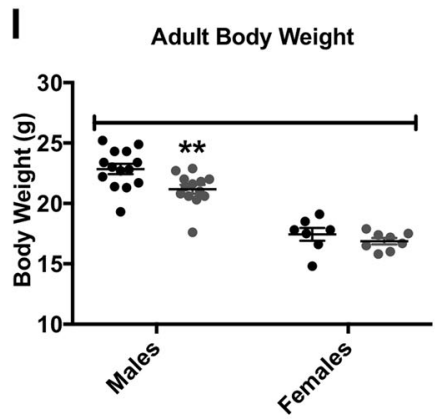

Fig. 1 Maternal IL-17A during gestation resulted in offspring somatic growth decrements but no gestational changes. a Experimental design [IHC (immunohistochemistry), qPCR (quantitative PCR), mRNA-seq (mRNA sequencing)]. b Maternal IL-17A increased maternal serum IL-17A, but not IL-6, and (c) trend-increased IL-17A in embryonic day 18 (E18) offspring kidney $(P=0.065)$ but (d) not placenta, all per ELISA ( $n=3$ saline, 4 IL-17 dams) ${ }^{\text {tr }} P<0.1,{ }^{*} P<0.05$ per two-sample $t$ test. e IL-17A did not change litter size (per Mann-Whitney) (f) or the number of resorptions at E18 (per Mann-Whitney) (four litters per condition). g Both male and female IL-17A offspring weighed less at E18 (main effect of condition via two-way ANOVA, $P<0.0001$; four litters per condition) and at (h) postnatal day 21 (P21) (main effect of condition, $P<0.005$; three litters per group except two litters for saline females), $\mathbf{i}$ but only males weighed less as adults (main effect of sex and condition via two-way ANOVA, $P<0.0001, P<0.05$, respectively; three litters per group except two litters for saline females). ${ }^{\operatorname{tr}} P<0.1,{ }^{*} P<0.05,{ }^{* *} P<0.01$ per Bonferroni's multiple comparisons test; brackets represent significant finding via ANOVA; error bars represent SEM.

Saline $=26 \pm 5 \%, \mathrm{IL}-17=32 \pm 3 \% ; P=0.27)$. Given smaller cortical volume with IL-17A, the increased $\mathrm{NeuN}+$ cell density was unremarkable. However, $\mathrm{S} 100 \beta+$ glial cells were decreased in density overall and also as a percent of all neocortical cells $[t(10)=$ $3.444, P=0.0063$ ] (Fig. $4 d-f)$, remarkable in a cortex also of smaller volume. Adult females showed none of these impacts (Supplementary Fig. 1A-D).

Decreased glial cell density was complemented by decreased glial gene expression in adult male neocortex (Fig. 4g). Nogo- $A$, Mobp, S100ß, Olig2, and Mag expression were decreased. Of synaptic genes decreased in male embryonic dorsal forebrain transcriptomics by maternal IL-17A (Fig. 2c), GABAergic, (Gabra1) but not glutamatergic (S/c17a8 and Grin2a), synapse-associated mRNA was increased in IL-17A condition adult males (Fig. 4h). Female neocortex had similarly increased Gabra1, but fewer and distinct changes in glial genes ( $\uparrow \mathrm{Ng} 2, \downarrow G F A P$; Supplementary Fig. 1).

Adult offspring behavioral effects of maternal IL-17A

IL-17A-condition adult males exhibited decreased anxiety-like behavior on the elevated plus maze [open/closed arm time: $t(22)=2.097, P=0.048 ; \%$ of time spent on open arm: $t(21)=$ 3.079, $P=0.006$; corrected alpha for $\%$ time $=0.025$ ] (Fig. 5a, b). Unlike males, female offspring anxiety-like behavior was trendwise increased [open/closed arm time: $t(12)=1.797, P=0.098 ; \%$ of time on open arm: $t(12)=1.789, P=0.099$; corrected alpha for $\%$ time $=0.025$ ] (Fig. 5e, f).

Open-field locomotor behavior was trend-wise increased among IL-17A condition males across both days [two-way ANOVA main effect of condition $F(1,24)=3.488, P=0.074]$ and trendwise increased on Day One of testing $[t(25)=2.271, P=0.032$; corrected alpha $=0.025$ ] but unchanged on day two (Fig. 5c). This day one activity reflected trend-wise abnormal intra-session habituation in male IL-17A condition offspring $[t(25)=1.789$, $P=0.086$ ] (Fig. 5d). Unlike males, open-field locomotor behavior across both days (Fig. $5 \mathrm{~g}$ ) and day one intra-session habituation (Fig. 5h) were unchanged in female offspring.

IL-17A males exhibited impaired sociability $[t(26)=2.436, P=$ 0.022] (Fig. 5i), which was both deficient relative to controls and, unlike controls, lacked a clear social preference [saline: $t(11)=2.348, P=0.039$; IL-17A: $t(15)=0.3811, P=0.708$ via one sample $t$ test vs $50 \%$ chance preference]. Interestingly, IL-17A 
A

E18 Male Dorsal Forebrain Volume

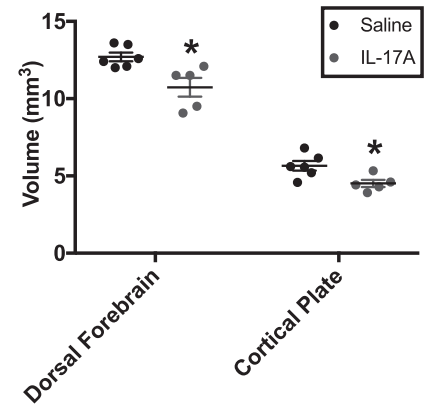

E E18 Female Dorsal Forebrain Volume
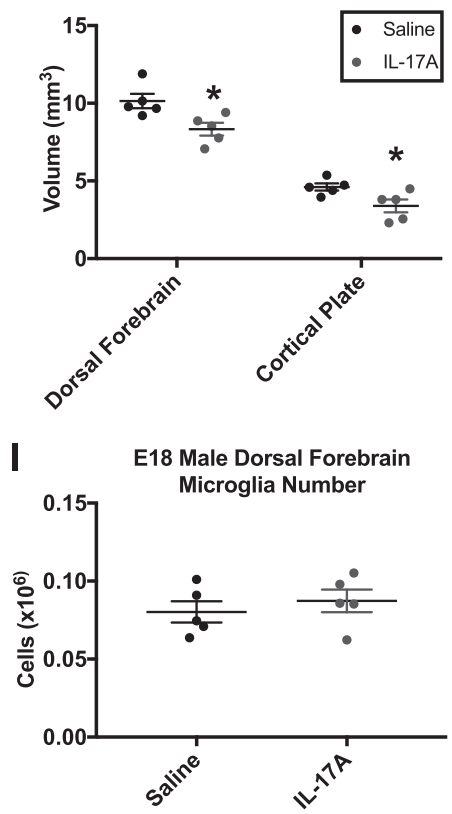

B E18 Male Cortical Plate \% Dorsal Forebrain

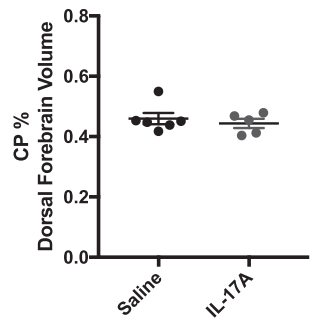

F E18 Female Cortical Plate $\%$ Dorsal Forebrain

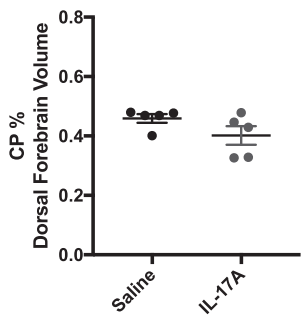

C E18 Male Dorsal Forebrain

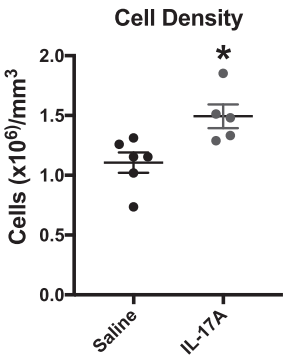

G E18 Female Dorsal Forebrain

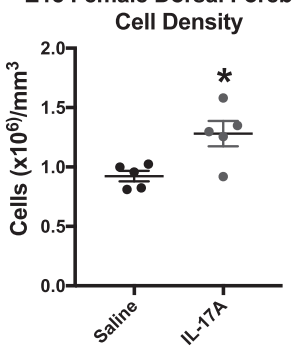

D E18 Male Dorsal Forebrain

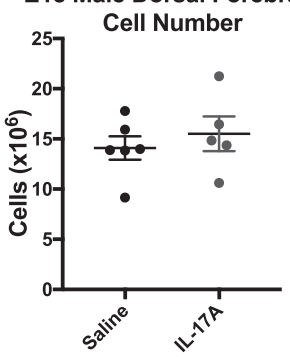

H E18 Female Dorsal Forebrain

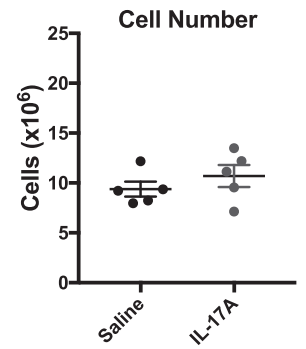

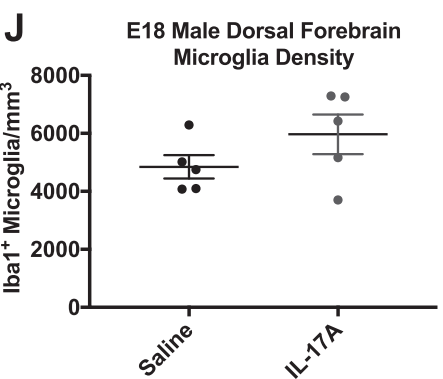

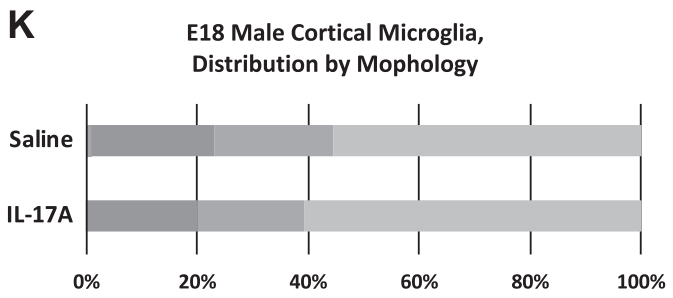

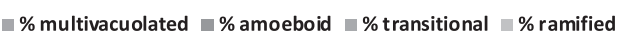

Fig. 2 Gross cortical development, but not embryonic microglia, was altered by maternal IL-17A. a Males exhibited decreased embryonic day 18 (E18) dorsal forebrain and cortical plate (CP) volumes (b), which were in proportion to one another. c Dorsal forebrain cell density by DAPI was significantly increased, such that (d) the total number of cells was unchanged. As in males, e females exhibited decreased E18 dorsal forebrain and CP volumes (f), which were in proportion to one another. $\mathbf{g}$ Dorsal forebrain cell density by DAPI was significantly increased, such that (h) the total number of cells was unchanged in female E18 pups exposed to maternal IL-17A. $\mathbf{i}$ The total number of dorsal forebrain microglia, $\mathbf{j}$ density of microglia, $\mathbf{k}$ and their distribution across morphologies was unchanged in E18 pups exposed to maternal IL-17A. To assess E18 $\mathrm{Iba}^{+}$dorsal forebrain microglia, four previously described categories [14] were used: (1) ramified, with a normal nucleus and three or more processes, (2) transitional, with a normal nucleus and one or two process, (3) amoeboid, with a normal nucleus and no processes, and (4) multivacuolated, with multiple, pyknotic nuclei and/or vacuoles. ( $n=3$ litters per condition with 1-2 offspring per litter for all measures) ${ }^{*} P<0.05$ per two-sample $t$ test, error bars represent SEM.

animals had more interactions across both cups [two-way ANOVA main effect of condition $F(1,55)=15.84, P=0.0002$; post hoc tests: empty, $t(28)=3.054, P=0.005$; social, $t(27)=2.577, P=0.016$, corrected alpha 0.025] (Fig. 5j), but their average time per interaction was different from controls [two-way ANOVA interaction $F(1,49)=5.875, P=0.0191]$. This was driven by a shorter empty cup time per interaction in IL-17A animals compared with controls [post hoc test: empty cup $t(25)=3.874, P=0.0007$; corrected alpha $=0.025]$ (Fig. 5k). Social preference was negatively correlated with Gabra5 expression (Fig. 5I) $[F(8)=$ 28.98, $R=-0.872 ; P<0.001$ by exploratory analyses; $q=0.004$ after Benjamini and Hochberg FDR correction]. There was no significant group-wise difference $(P>0.05)$ in total distance traveled in either the social (saline: $51.31 \pm 1.40 \mathrm{~m}$; IL-17A: 47.46 $\pm 2.13 \mathrm{~m}$ ) or elevated plus maze (saline: $12.3 \pm 0.62 \mathrm{~m}$; IL-17A: $13.91 \pm 0.56 \mathrm{~m}$ ) assays.
Neither male nor female offspring exhibited altered anxietylike behavior on center time in the open field (Supplementary Fig. 3A, B). Likewise, sensorimotor gating was unchanged by maternal IL-17A in male and female offspring (Supplementary Fig. 3C, D).

\section{DISCUSSION}

Given high rates of ASD diagnosis and associated public health costs, identification of treatment and prevention targets for this disorder is critically important. Here, we demonstrate that chronic maternal IL-17A during murine gestation caused neurodevelopmental and behavioral phenotypes relevant to ASD. Notably, embryonic dorsal forebrain exhibited male-specific altered expression of synapse-, cell cycle-, and ASD-associated genes. This was accompanied in adulthood by decreased neocortical glia, altered 
A

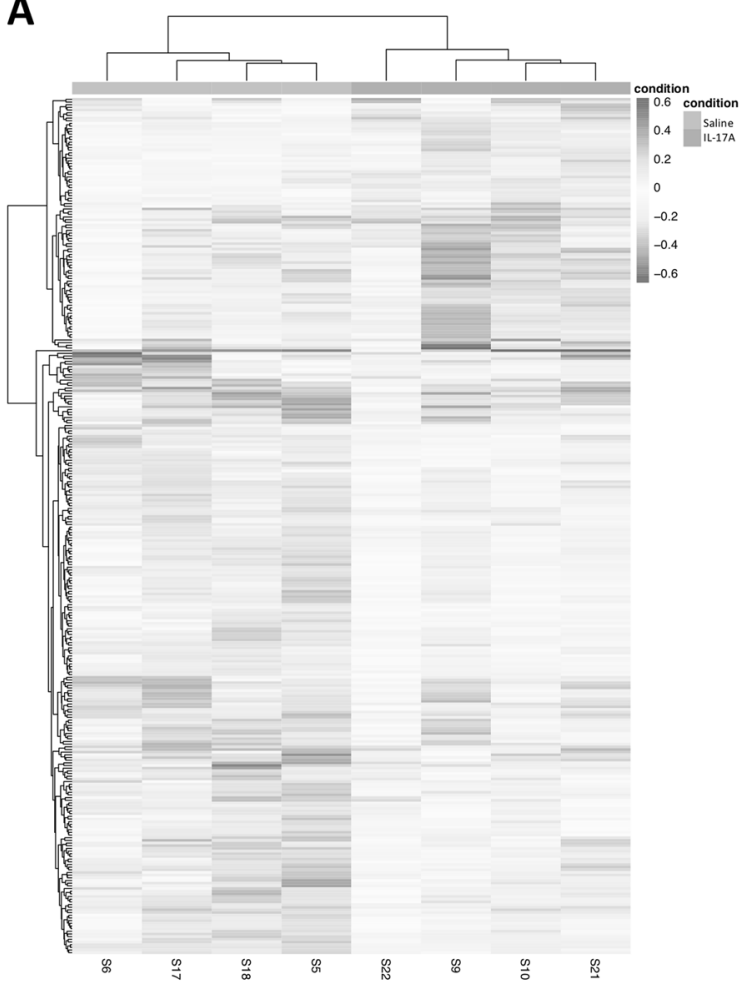

E

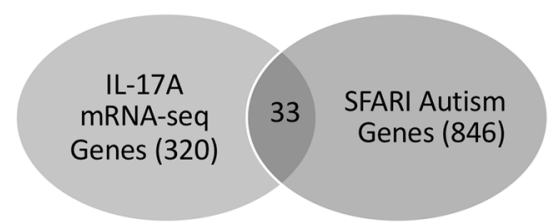

B
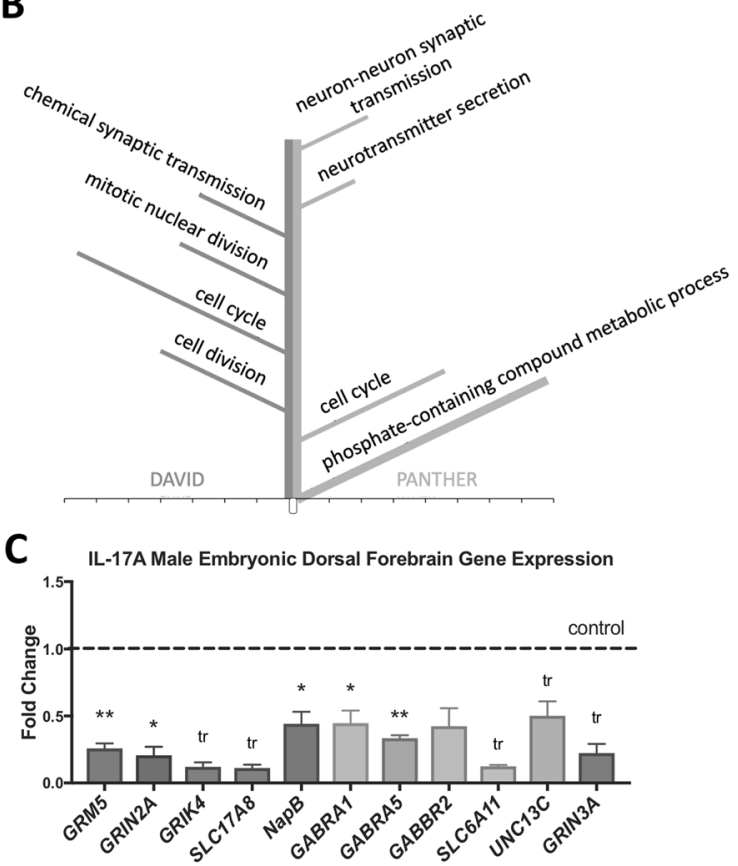

D IL-17A Female Embryonic Dorsal Forebrain Gene Expression

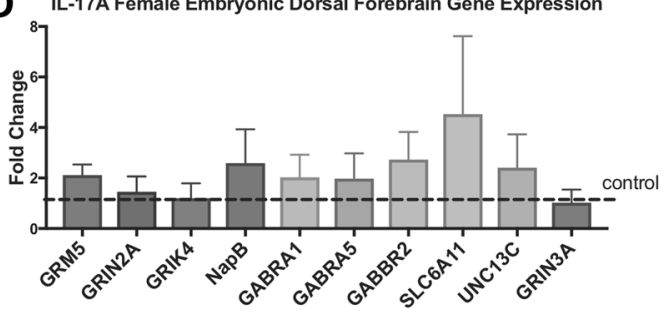

Fig. 3 Maternal IL-17A altered male but not female offspring embryonic cortex transcriptome. a Heatplot representing 320 genes that were differentially expressed in dorsolateral cortex of male IL-17A-condition offspring (none changed in females) per mRNA sequencing (mRNA-seq) ( $n=4$; one animal from each of four litters per condition). $\mathbf{b}$ The results of Gene Ontology (GO) Enrichment analysis of the 320 differentially expressed genes, depicted as previously [24]. $x$-axis: the number of genes in each pathway; $y$-axis: extent of enrichment; thickness: $P$-value $(P<0.05)$; GO terms for biological processes derived from the Database for Annotation, Visualization, and Integrated Discovery (DAVID, left, blue); GO terms for biological processes derived from Protein ANalysis THrough Evolutionary Relationships Classification System (PANTHER, right, green). c Genes from the biological process pathway with the highest fold enrichment ("neuron-neuron synaptic transmission") were assessed in male tissues for decrements with IL-17A, as predicted by mRNA-seq, via qPCR ( $n=4$ saline, 3 IL-17A). d These same genes were unchanged via pPCR in female tissues (S/c17a8 excluded due to poor amplification in female samples, $n=3$ saline, 4 IL-17A). $y$-axis represents expression relative to control levels, with $1=$ control level. e Simons Foundation for Autism Research Initiative (SFARI) genes were significantly overrepresented in IL-17A male offspring mRNA-seq data $(P=4.83 \mathrm{E}-11$, Fisher's test). ${ }^{t r} P<0.1,{ }^{*} P<0.05,{ }^{* *} P<0.005$ per two-sample $t$ test, error bars represent SEM.

synaptic and glial genes, and altered social, anxiety-like, and habituation behaviors in males.

We identified fetal growth restriction in this model, a finding echoed in clinical autism literature [25] and preclinical short-term MIA models [26, 27]. This growth restriction was symmetrical (brain and body) and persistent (fetal and adult), unlike asymmetrical, brain-sparing growth restriction in inflammatory pregnancy conditions (preeclampsia, diabetes) $[28,29]$. This may reflect contributions from non-IL-17A mechanisms. Total brain and cortical growth alterations also occur in individuals with ASD [30].

Prenatally, IL-17A may influence neurodevelopment via indirect action on maternal physiology or direct action on neurons. For instance, IL-17 mechanisms alter cell differentiation, survival, and signaling [31, 32], and IL-17A regulates adult hippocampal neurogenesis, dentate gyrus synaptic excitability, and hippocampal pro- and anti-inflammatory cytokines [33]. MIA and IL-17 activate microglia [34], which phagocytose neural progenitors. However, we found no evidence of increased embryonic microglial phagocytic morphologies here. Further, our data suggest that reduced embryonic dorsal forebrain size was not due to reduced total cell number, despite evidence that IL-17 decreases neural stem cell proliferation in vitro [35]. Nonmorphological effects of maternal IL-17A on offspring microglia is an important remaining question.

The transcriptomic findings described here provide a link between chronic maternal IL-17 and ASD-relevant phenotypes, including a significant role for altered synaptic processes [36]. We report overrepresentation of ASD-linked genes and transcriptional dysregulation of GABA receptor subunit genes Gabra1 and Gabra5, among others, in male embryonic dorsal forebrain after maternal IL-17A. These findings may reflect a suppression of late gestation neurodevelopmental processes, including normal upregulation of synapse-related genes. Late gestation proliferation of cortical progenitors produce glial cells. The overrepresentation of cell cycle genes among those changed by IL-17A may reflect an influence on glial cell production.

In line with impaired early processes, male adult neocortical S100ß-labeled cells and expression of multiple glial genes were 
A

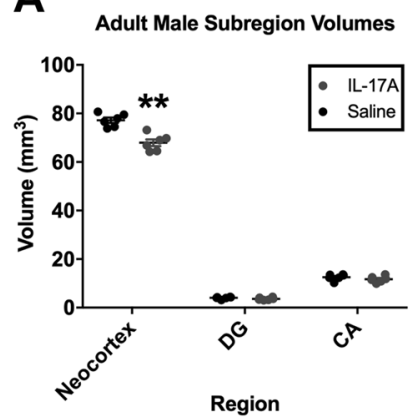

B

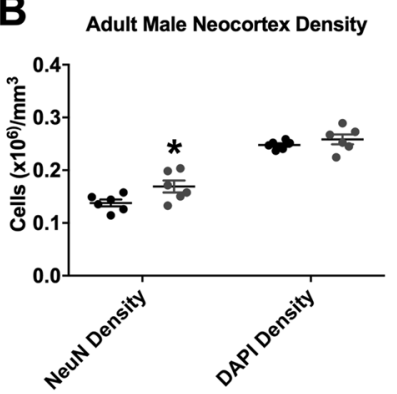

C

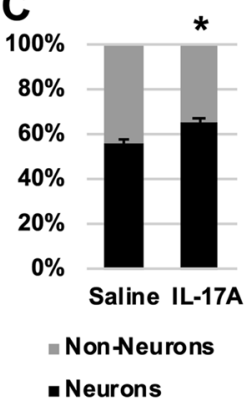

G

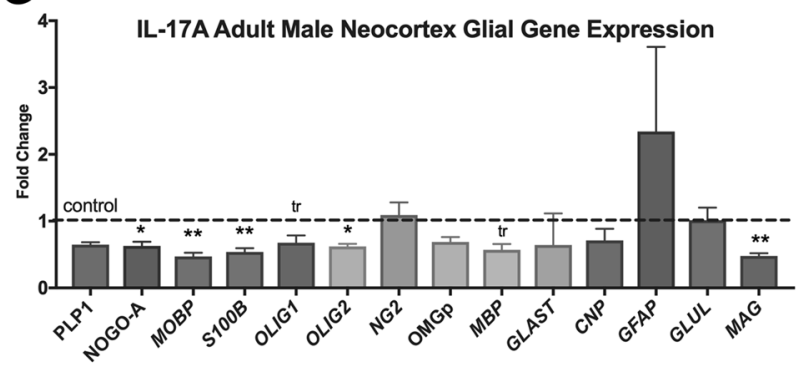

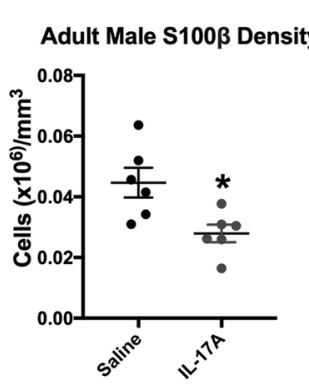

E

F

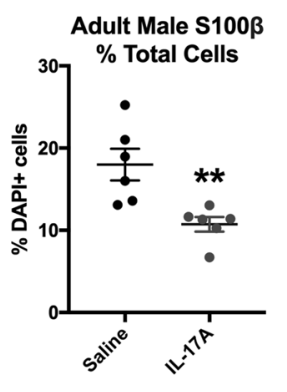

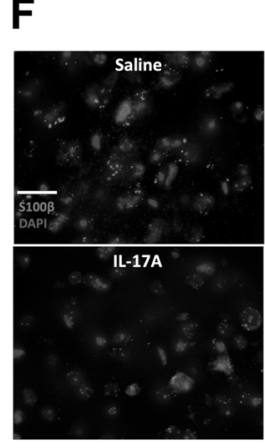

H

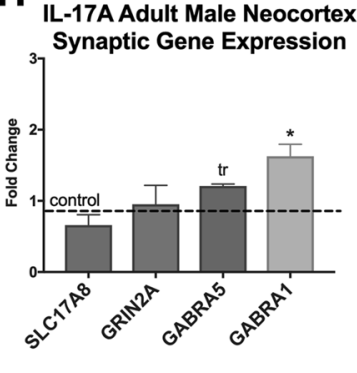

Fig. 4 Adult offspring neocortex was altered by maternal IL-17A. a IL-17A adult male offspring had smaller neocortical volumes, but unchanged hippocampal dentate gyrus (DG) or CA volumes. b IL-17A adult males also exhibited a significantly increased density of NeuN ${ }^{+}$ neurons, but unchanged total nuclei (DAPI +) density in the neocortex. This led to (c) a shift in the composition of the neocortex, with IL-17A animals exhibiting a greater percent of neurons than controls. Non-neuron percentages are derived from neuron percentages. $\mathbf{d}$ IL-17A adult males exhibited a significantly decreased density of $\mathrm{S} 100 \beta^{+}$cells, which (e) comprised a decreased \% of total cells. f Micrographs depicting anti-S100 $\beta$ immunohistochemistry in the neocortex. g Assessment of neocortical glial gene expression by qPCR revealed widespread decreases ( $n=3$ saline, four IL-17A, each from a separate litter). $\mathbf{h}$ qPCR revealed increased expression of Gabra5 and Gabra1, but not of S/c17a8 and Grin2 $a$ in the neocortex. $y$-axes (panels $\mathbf{g}, \mathbf{h}$ ) represent expression relative to control levels. ${ }^{\operatorname{tr}} P<0.1,{ }^{*} P<0.05,{ }^{* *} P<0.01$ per two-sample $t$ test, scale bar (panel $\mathbf{f})=10 \mu \mathrm{m}$, error bars represent SEM.

decreased after chronic maternal IL-17A, indicating oligodendrocyte and/or astrocyte deficits. Glial dysfunctions, including altered myelination, white matter tractography, organization, and connectivity, have been noted in ASD cortex [37] across development [38]. In addition, blood $S 100 \beta$ levels, which may indicate glial damage, are increased in individuals with ASD and with ASD severity [39]. MIA and acute exposure to IL-17 in vivo increased GFAP expression in reactive astrocytes in the amygdala and spinal cord [40, 41]. However, we found no evidence for increased GFAP in adult neocortex. Rather, elevated fetal IL-17 itself may alter production or differentiation of cortical glia. Direct effects of IL-17 in vitro include reduced neural progenitor differentiation into glial subtypes [35]. Future studies should explore glial cell subtypes and white matter tractography after prenatal maternal IL-17 or chronic inflammation.

Reduced mature glia may secondarily influence neocortical synapses [42]. As we found in adult neocortex, $G_{A B A}$ receptor expression is also increased in Polyl:C MIA adult offspring hippocampus and amygdala [43]. PET imaging of individuals with ASD indicates reduced Gabra5 binding in multiple brain regions (e.g., amygdala, nucleus accumbens) [44]. Gabra5 deletion in mice results in altered sociability [45], and Gabra1 and Gabra5 protein levels are downregulated in ASD brain [46]. These results overlap with our finding of correlated neocortical $G A B A_{A}$ receptor expression and sociability. $G_{A B A_{A}}$ receptor changes are likely heterogenous within ASD, with some driven transcriptionally and some post-translationally [47].

Male but not female IL-17A offspring dorsal forebrain transcriptome was enriched for ASD-related genes in the present study, reflecting the male bias in ASD [roughly 4:1 (CDC)]. This male predominance differs from studies reporting greater IL-17 reactivity in the presence of female hormones $[48,49]$ and may arise from our focus on early development, preceding fetal sex hormone production. In early development, placental sex differences influence the brain [50]. Future work should clarify the sex-specific role of IL-17 in embryonic neurodevelopment, including placental mediators.

Our behavioral findings overlap with both the MIA and ASD literature. IL-17A males exhibited altered social and anxiety-like behaviors. Social deficits result from short-term MIA exposure and may occur via prenatal IL-17-mediated mechanisms $[9,51]$. The occurrence of reduced male sociability despite decreased anxiety was a meaningful dissociation of these intersecting functional domains. Dysregulations of interaction number and time spent in each interaction may be components of this reduced sociability. Females did not show decreased anxiety-like behavior, but were not tested for sociability, a limitation of this study. Notably, MIA typically leads to increased [52, 53] not decreased anxiety in males, as found here. This difference may be driven by long-term, lower dose immune perturbation, or the specificity of maternal IL17 cytokine exposure, utilized here. Of interest, gestational IL-17A levels are also positively correlated with child cognitive performance [54], suggesting a complex role for this cytokine.

Our approach did not account for potential postnatal influences of maternal inflammation. MIA during pregnancy alters maternal nest-building and pup retrieval [55], which may alter offspring neurodevelopment. Furthermore, dams were singly housed here as previously $[53,56,57]$, a potential source of interacting stress. Group-housing of pregnant dams and cross-fostering have limitations [58], but could distinguish these impacts in future studies.

IL-17 may act at multiple levels during chronic pregnancy infusion-maternal, placental, and fetal brain physiology may be disrupted. Maternal IL-17 dysregulates blood pressure and redox 

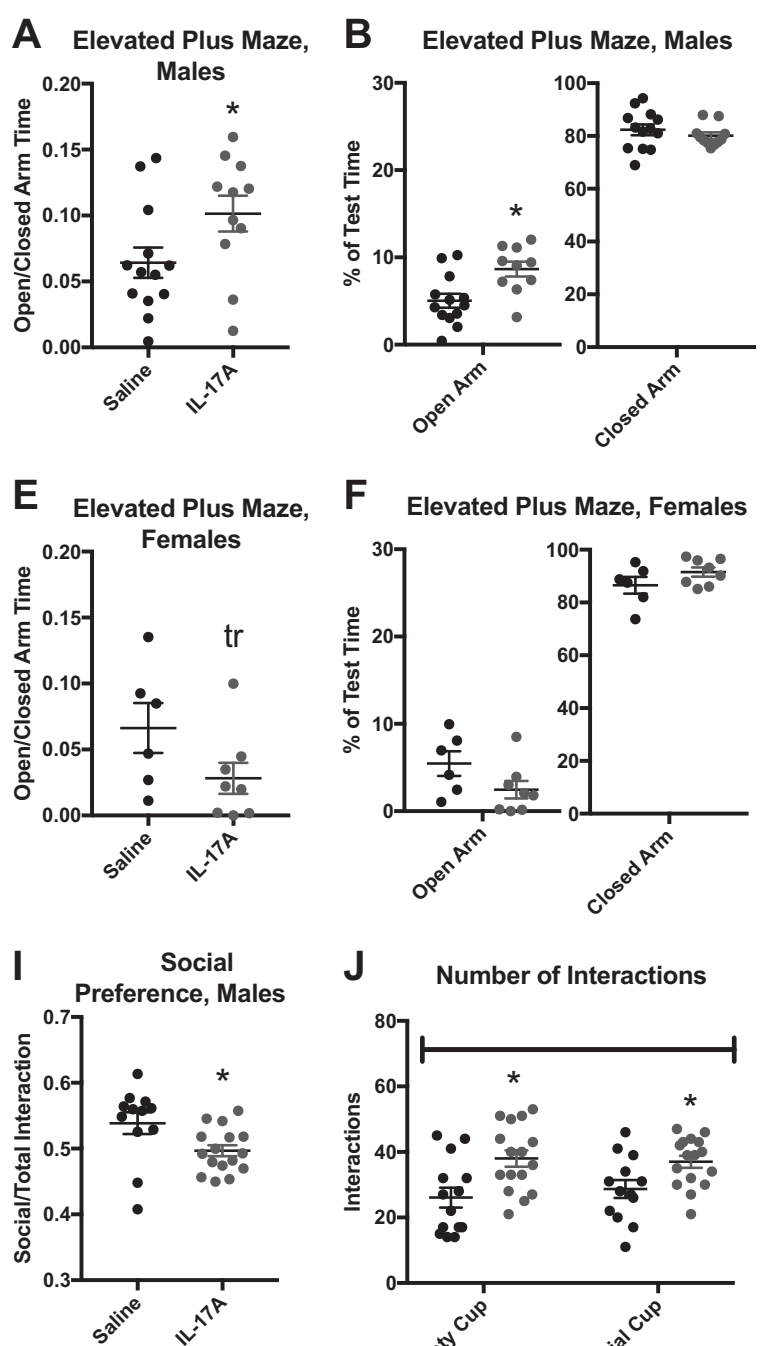

J Number of Interactions

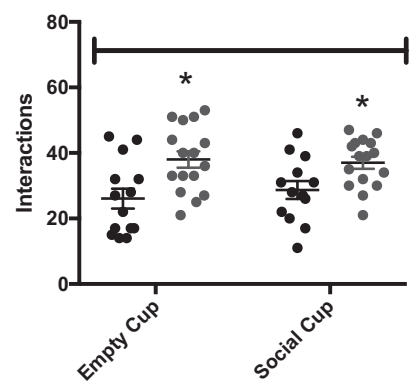

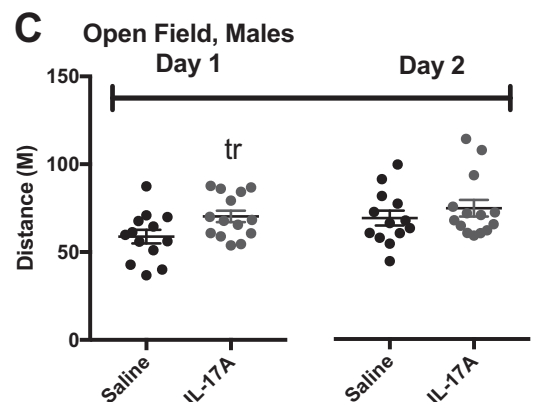

D Day 1 Open Field Habituation, Males

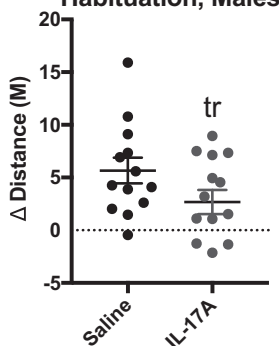

G
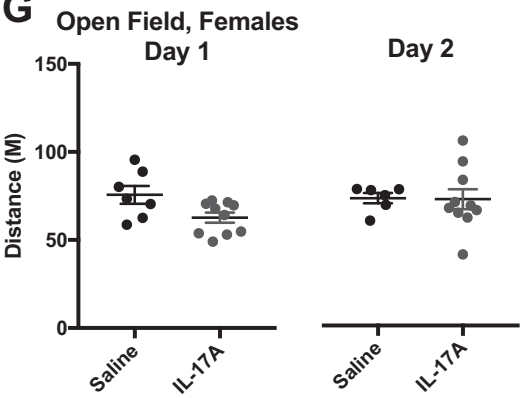

H Day 1 Open Field Habituation, Females

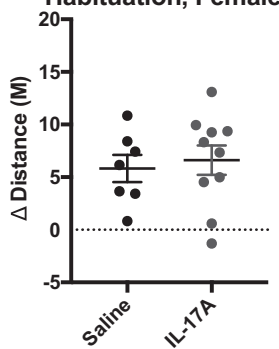

K

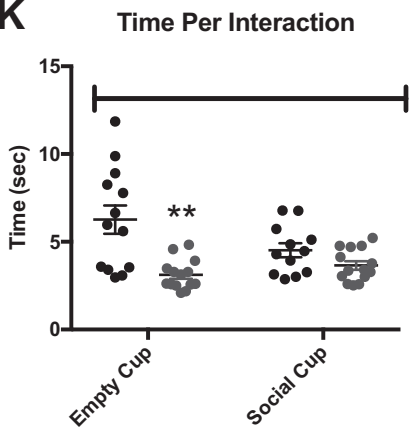

$\mathbf{L}$

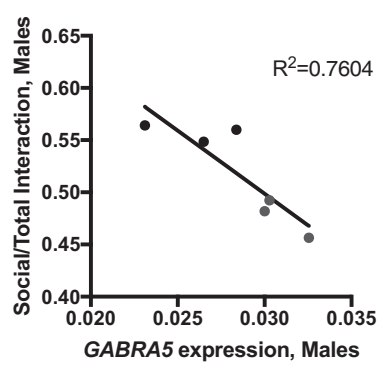

Fig. 5 Adult offspring behavior sex-specifically changed by embryonic maternal IL-17A. a IL-17A adult males exhibited decreased anxietylike behavior on the elevated plus maze (b), including significantly increased time spent on the open arm. c Locomotor behavior was trendwise increased across both days by repeated measures two-way ANOVA [main effect of day $(P=0.029)$; trend main effect of condition $(P=$ $0.074)$ ], specifically in IL-17A males on day one (corrected alpha $=0.025)$. Increased day one locomotion was explained by $(\mathbf{d})$ trend-wise impaired day one open-field intra-session habituation (difference between distance traveled during first and last 5 min) in IL-17A adult males. e IL-17A adult females exhibited a trend increase in anxiety-like behavior on the elevated plus maze and (f) no change in percent time in open or closed arms. In female offspring, $\mathbf{g}$ locomotor behavior did not differ (no significant interaction or main effects). $\mathbf{h}$ Day one open-field habituation was unchanged in IL-17A adult females. $\mathbf{i}$ IL-17A condition adult males also exhibited decreased social preference in the threechambered social preference task $(P=0.022)(j)$ and increased total interactions with either cup [main effect of condition $(P=0.0002)$; post hoc tests: empty cup $(P=0.005)$, social cup $(P=0.016)$; corrected alpha $=0.025]$. $\mathbf{k} \mathrm{IL}-17 \mathrm{~A}$ condition adult males spent less time per interaction with the empty cup [interaction $(P=0.0191)$; post hoc test: empty cup $(P=0.0007)$; corrected alpha $=0.025$ ]. I A significant negative correlation was found between social preference behavior and neocortical Gabra5 expression $(R=-0.872 ; P<0.05$ by exploratory analyses; $q$ $=0.004$ after Benjamini and Hochberg FDR correction). ${ }^{\operatorname{tr}} P<0.1,{ }^{*} P<0.05,{ }^{*} P<0.005$ per two-sample $t$ test, error bars represent SEM, $n=$ 2-3 saline litters; four IL-17A litters, with 1-4 animals per litter.

balance [59], as well as mediates renal and vascular inflammation and function [60], maternal factors that may independently regulate offspring nutrient transfer, growth, and development. Placental processes including angiogenesis [61] and trophoblast invasion, proliferation, and apoptosis [62] are also regulated by IL17. Finally, IL-17 may cross the placenta and bind to developing brain tissues before blood brain barrier formation (approximately E15 in mice), initiating downstream intracellular NF-KB and ERK mechanisms, among others $[9,31]$. These mechanisms could directly alter synaptic gene expression (e.g., Grin2a, which has an NF-KB binding site in its promoter) and/or broader signaling cascades (e.g., ERK signaling, which agonizes GABA systems) [63, 64]. Trans-placental IL-17 passage has not been shown directly (e.g., with radiotracer studies), but maternal IL-17 may increase fetal IL-17, as a trend in fetal kidney here, without direct passage.

While this study suggests intriguing links between chronic maternal IL-17 and offspring ASD-relevant neurodevelopmental trajectories, it does not demonstrate that a single cytokine drives ASD risk. In fact, as we have argued previously [3], prenatal challenges likely trigger broad, secretome-level inflammatory changes that alter neurodevelopmental landscapes. While prior work has implicated elevated fetal brain IL-17 in deleterious outcomes, this study points to a significant role for maternal IL-17, a more accessible and similarly high-yield target. Furthermore, this study shines a light on the potential impact of IL-17 mechanisms 
in persistent inflammation during pregnancy and neurodevelopmental consequences, as in preeclampsia and gestational diabetes.

In summary, we found that chronic gestational IL-17 mechanisms underlie ASD-related phenotypes in mice: a smaller cortex with altered synaptic and cell cycle gene expression prenatally; disrupted behaviors and glial neurobiology in adulthood. Identification of the neural substrates and specific factors underlying neurodevelopmental disorders is critical for developing therapies. This work suggests that targeting canonical and noncanonical IL17 pathways may promote prevention and/or treatment of ASD and related psychopathologies. Microbiome, epigenetic, or lipidomic strategies $[65,66]$ and direct IL-17-attenuating medical therapy (e.g., monoclonal antibodies) [67] are promising.

\section{FUNDING AND DISCLOSURE}

This work was supported by the University of lowa Center for Hypertension Research Pilot Grant Program; the lowa Center for Research by Undergraduates; T32MH019113, the postdoctoral lowa Neuroscience Specialty Program in Research Education (INSPIRE); and a University of lowa DeLTA Center Research Grant. Some data were obtained at the Neural Circuits and Behavior Core at the lowa Neuroscience Institute, supported in part by the Roy J. Carver Charitable Trust. The authors declare no conflict of interest.

\section{ACKNOWLEDGEMENTS}

The authors wish to acknowledge the University of lowa Genomics Core; Dr. Donna Santillan for her immunology expertise; and Stevens lab members for helpful discussion. The authors also wish to acknowledge Christoph Dotter (Novarino Lab) for his help with depicting gene ontology pathway changes.

\section{ADDITIONAL INFORMATION}

Supplementary Information accompanies this paper at (https://doi.org/10.1038/ s41386-020-0640-0)

Publisher's note Springer Nature remains neutral with regard to jurisdictional claims in published maps and institutional affiliations.

\section{REFERENCES}

1. Mann JR, McDermott S, Bao H, Hardin J, Gregg A. Pre-eclampsia, birth weight, and autism spectrum disorders. J Autism Dev Disord. 2010;40:548-54.

2. Xiang $\mathrm{AH}$. Association of maternal diabetes with autism in offspring. JAMA 2015;317:537-8.

3. Gumusoglu SB, Stevens HE. Maternal inflammation and neurodevelopmental programming: a review of preclinical outcomes and implications for translational psychiatry. Biol Psychiatry. 2019;85:107-21

4. Scroggins SM, Santillan DA, Lund JM, Sandgren JA, Krotz LK, Hamilton WS, et al. Elevated vasopressin in pregnant mice induces T-helper subset alterations consistent with human preeclampsia. Clin Sci. 2018;132:419-36.

5. Molvarec A, Czegle I, Szijarto J, Rigo J Jr. Increased circulating interleukin-17 levels in preeclampsia. J Reprod Immunol. 2015;112:53-7.

6. Cao W, Wang X, Chen T, Xu W, Feng F, Zhao S, et al. Maternal lipids, BMI and IL$17 / \mathrm{IL}-35$ imbalance in concurrent gestational diabetes mellitus and preeclampsia. Exp Ther Med. 2018;16:427-35.

7. Akintunde $M E$, Rose $M$, Krakowiak $P$, Heuer $L$, Ashwood $P$, Hansen $R$, et al. Increased production of IL-17 in children with autism spectrum disorders and comorbid asthma. J Neuroimmunol. 2015;286:33-41.

8. van der Zwaag B, Franke L, Poot M, Hochstenbach R, Spierenburg HA, Vorstman $J A$, et al. Gene-network analysis identifies susceptibility genes related to glycobiology in autism. PLoS ONE. 2009;4:e5324.

9. Choi GB, Yim YS, Wong H, Kim S, Kim H, Kim SV, et al. The maternal interleukin17a pathway in mice promotes autism-like phenotypes in offspring. Science. 2016;351:933-9.

10. Kentner AC, Bilbo SD, Brown AS, Hsiao EY, McAllister AK, Meyer U, et al. Maternal immune activation: reporting guidelines to improve the rigor, reproducibility, and transparency of the model. Neuropsychopharmacology. 2019;44:245-58.
11. Harrington AJ, Raissi A, Rajkovich K, Berto S, Kumar J, Molinaro G, et al. MEF2C regulates cortical inhibitory and excitatory synapses and behaviors relevant to neurodevelopmental disorders. eLife. 2016;5:e20059.

12. Bagot RC, Cates HM, Purushothaman I, Lorsch ZS, Walker DM, Wang J, et al. Circuit-wide transcriptional profiling reveals brain region-specific gene networks regulating depression susceptibility. Neuron. 2016;90:969-83.

13. Zhang ZH, Jhaveri DJ, Marshall VM, Bauer DC, Edson J, Narayanan RK, et al. A comparative study of techniques for differential expression analysis on RNA-Seq data. PLoS ONE. 2014;9:e103207.

14. Gumusoglu SB, Fine RS, Murray SJ, Bittle JL, Stevens HE. The role of IL-6 in neurodevelopment after prenatal stress. Brain Behav Immun. 2017;65:274-83.

15. Stevens HE, Smith KM, Maragnoli ME, Fagel D, Borok E, Shanabrough M, et al. Fgfr2 is required for the development of the medial prefrontal cortex and its connections with limbic circuits. J Neurosci. 2010;30:5590-602.

16. Bray NL, Pimentel H, Melsted P, Pachter L. Near-optimal probabilistic RNA-seq quantification. Nat Biotechnol. 2016;34:525-7.

17. Zhang $C$, Zhang B, Lin LL, Zhao S. Evaluation and comparison of computational tools for RNA-seq isoform quantification. BMC Genomics. 2017;18:583.

18. Jin $\mathrm{H}$, Wan YW, Liu Z. Comprehensive evaluation of RNA-seq quantification methods for linearity. BMC Bioinform. 2017;18(Suppl 4):117.

19. Pimentel H, Bray NL, Puente S, Melsted P, Pachter L. Differential analysis of RNAseq incorporating quantification uncertainty. Nat Methods. 2017;14:687-90.

20. Costa-Silva J, Domingues D, Lopes FM. RNA-Seq differential expression analysis: an extended review and a software tool. PLoS ONE. 2017;12:e0190152.

21. Mi H, Huang X, Muruganujan A, Tang H, Mills C, Kang D, et al. PANTHER version 11: expanded annotation data from Gene Ontology and Reactome pathways, and data analysis tool enhancements. Nucleic Acids Res. 2017;45(D1):D183-D89.

22. Huang dW, Sherman BT, Lempicki RA. Bioinformatics enrichment tools: paths toward the comprehensive functional analysis of large gene lists. Nucleic Acids Res. 2009;37:1-13.

23. Huang dW, Sherman BT, Lempicki RA. Systematic and integrative analysis of large gene lists using DAVID bioinformatics resources. Nat Protoc. 2009;4:44-57.

24. Tărlungeanu DC, Deliu E, Dotter CP, Kara M, Janiesch PC, Scalise $M$, et al. Impaired amino acid transport at the blood brain barrier is a cause of autism spectrum disorder. Cell. 2016;167:1481-94.e18.

25. Abel KM, Dalman C, Svensson AC, Susser E, Dal H, Idring S, et al. Deviance in fetal growth and risk of autism spectrum disorder. Am J Psychiatry. 2013;170:391-8.

26. Girardi G, Yarilin D, Thurman JM, Holers VM, Salmon JE. Complement activation induces dysregulation of angiogenic factors and causes fetal rejection and growth restriction. J Exp Med. 2006;203:2165-75.

27. Xu DX, Chen YH, Wang H, Zhao L, Wang JP, Wei W. Tumor necrosis factor alpha partially contributes to lipopolysaccharide-induced intra-uterine fetal growth restriction and skeletal development retardation in mice. Toxicol Lett. 2006;163:20-9.

28. Rasmussen S, Irgens LM. Fetal growth and body proportion in preeclampsia Obstet Gynecol. 2003;101:575-83.

29. Reece EA, Smikle C, O'Connor TZ, Holford T, Nelson-Robinson L, Degennaro N, et al. A longitudinal study comparing growth in diabetic pregnancies with growth in normal gestations: I. the fetal weight. Obstet Gynecol Surv. 1990;45:161-4.

30. Fombonne E, Roge B, Claverie J, Courty S, Fremolle J. Microcephaly and macrocephaly in autism. J Autism Dev Disord. 1999;29:113-9.

31. Wong H, Hoeffer C. Maternal IL-17A in autism. Exp Neurol. 2018;299(Pt A):228-40.

32. Bolte AC, Lukens JR. Th17 cells in Parkinson's disease: the bane of the midbrain. Cell Stem Cell. 2018;23:5-6.

33. Liu Q, Xin W, He P, Turner D, Yin J, Gan Y, et al. Interleukin-17 inhibits adult hippocampal neurogenesis. Sci Rep. 2014;4:7554.

34. Cunningham CL, Martinez-Cerdeno V, Noctor SC. Microglia regulate the number of neural precursor cells in the developing cerebral cortex. J Neurosci. 2013;33:4216-33.

35. Li Z, Li K, Zhu L, Kan Q, Yan Y, Kumar P, et al. Inhibitory effect of IL-17 on neural stem cell proliferation and neural cell differentiation. BMC Immunol. 2013;14:20.

36. Schwede M, Nagpal S, Gandal MJ, Parikshak NN, Mirnics K, Geschwind DH, et al. Strong correlation of downregulated genes related to synaptic transmission and mitochondria in post-mortem autism cerebral cortex. J Neurodev Disord. 2018;10:18.

37. Shukla DK, Keehn B, Muller RA. Tract-specific analyses of diffusion tensor imaging show widespread white matter compromise in autism spectrum disorder. J Child Psychol Psychiatry. 2011;52:286-95.

38. Ameis SH, Catani M. Altered white matter connectivity as a neural substrate for social impairment in autism spectrum disorder. Cortex. 2015;62:158-81.

39. Al-Ayadhi LY, Mostafa GA. A lack of association between elevated serum levels of $\mathrm{S} 100 \mathrm{~B}$ protein and autoimmunity in autistic children. J Neuroinflammation. 2012;9:54. 
40. You T, Bi Y, Li J, Zhang M, Chen X, Zhang K. IL-17 induces reactive astrocytes and up-regulation of vascular endothelial growth factor (VEGF) through JAK/STAT signaling. Sci Rep. 2017;7:41779.

41. O'Loughlin E, Pakan JMP, Yilmazer-Hanke D, McDermott KW. Acute in utero exposure to lipopolysaccharide induces inflammation in the pre- and postnatal brain and alters the glial cytoarchitecture in the developing amygdala. J Neuroinflammation. 2017;14:212.

42. Chung WS, Welsh CA, Barres BA, Stevens B. Do glia drive synaptic and cognitive impairment in disease? Nat Neurosci. 2015;18:1539-45.

43. Nyffeler M, Meyer U, Yee BK, Feldon J, Knuesel I. Maternal immune activation during pregnancy increases limbic GABAA receptor immunoreactivity in the adult offspring: implications for schizophrenia. Neuroscience. 2006;143:51-62.

44. Mendez MA, Horder J, Myers J, Coghlan S, Stokes P, Erritzoe D, et al. The brain GABA-benzodiazepine receptor alpha- 5 subtype in autism spectrum disorder: a pilot [(11)C]Ro15-4513 positron emission tomography study. Neuropharmacology. 2013;68:195-201.

45. Zurek AA, Kemp SW, Aga Z, Walker S, Milenkovic M, Ramsey AJ, et al. alpha5GABAA receptor deficiency causes autism-like behaviors. Ann Clin Transl Neurol. 2016;3:392-8.

46. Fatemi SH, Reutiman TJ, Folsom TD, Thuras PD. GABA(A) receptor downregulation in brains of subjects with autism. J Autism Dev Disord. 2009;39:223-30.

47. Crider A, Pandya CD, Peter D, Ahmed AO, Pillai A. Ubiquitin-proteasome dependent degradation of GABAAalpha1 in autism spectrum disorder. Mol Autism. 2014;5:45.

48. Khan D, Dai R, Karpuzoglu E, Ahmed SA. Estrogen increases, whereas IL-27 and IFN-gamma decrease, splenocyte IL-17 production in WT mice. Eur J Immunol. 2010;40:2549-56.

49. Gilliver SC. Sex steroids as inflammatory regulators. J Steroid Biochem Mol Biol. 2010;120:105-15.

50. Bale TL. The placenta and neurodevelopment: sex differences in prenatal vulnerability. Dialogues Clin Neurosci. 2016;18:459-64.

51. Gumusoglu SB, Stevens HE Maternal inflammation and neurodevelopmental programming: a review of preclinical outcomes and implications for translational psychiatry. Biol. Psychiatry. 2019;85:107-21.

52. Depino AM. Early prenatal exposure to LPS results in anxiety- and depressionrelated behaviors in adulthood. Neuroscience. 2015;299:56-65.

53. Hao K, Su X, Luo B, Cai $Y$, Chen $T$, Yang $Y$, et al. Prenatal immune activation induces age-related alterations in rat offspring: effects upon NMDA receptors and behaviors. Behav Brain Res. 2019;370:111946.

54. Dozmorov MG, Bilbo SD, Kollins SH, Zucker N, Do EK, Schechter JC, et al. Associations between maternal cytokine levels during gestation and measures of child cognitive abilities and executive functioning. Brain Behav Immun. 2018;70:390-7.
55. Aubert A, Goodall G, Dantzer R, Gheusi G. Differential effects of lipopolysaccharide on pup retrieving and nest building in lactating mice. Brain Behav Immun. 1997;11:107-18.

56. Pendyala G, Chou S, Jung Y, Coiro P, Spartz E, Padmashri R, et al. Maternal Immune activation causes behavioral impairments and altered cerebellar cytokine and synaptic protein expression. Neuropsychopharmacology. 2017;42:1435-46.

57. Osborne AL, Solowij N, Babic I, Huang XF, Weston-Green K. Improved social interaction, recognition and working memory with cannabidiol treatment in a prenatal infection (poly $\mathrm{I}: \mathrm{C}$ ) rat model. Neuropsychopharmacology. 2017:42:1447-57.

58. Howells FM, Bindewald L, Russell VA. Cross-fostering does not alter the neurochemistry or behavior of spontaneously hypertensive rats. Behav Brain Funct. 2009;5:24

59. Dhillion $P$, Wallace $K$, Herse F, Scott J, Wallukat G, Heath J, et al. IL-17-mediated oxidative stress is an important stimulator of AT1-AA and hypertension during pregnancy. Am J Physiol Regul Integr Comp Physiol. 2012;303:R353-8.

60. Saleh MA, Norlander AE, Madhur MS. Inhibition of interleukin 17-A but not interleukin-17F signaling lowers blood pressure and reduces end-organ inflammation in angiotensin II-induced hypertension. JACC Basic Transl Sci. 2016;1:606-16.

61. Nadkarni S, Smith J, Sferruzzi-Perri AN, Ledwozyw A, Kishore M, Haas R, et al. Neutrophils induce proangiogenic $T$ cells with a regulatory phenotype in pregnancy. Proc Natl Acad Sci USA. 2016;113:E8415-E24.

62. Wu HX, Jin LP, Xu B, Liang SS, Li DJ. Decidual stromal cells recruit Th17 cells into decidua to promote proliferation and invasion of human trophoblast cells by secreting IL-17. Cell Mol Immunol. 2014;11:253-62.

63. Richter $M$, Suau $P$, Ponte I. Sequence and analysis of the $5^{\prime}$ flanking and $5^{\prime}$ untranslated regions of the rat N-methyl-D-aspartate receptor $2 \mathrm{~A}$ gene. Gene. 2002;295:135-42.

64. Cui Y, Costa RM, Murphy GG, Elgersma Y, Zhu Y, Gutmann DH, et al. Neurofibromin regulation of ERK signaling modulates GABA release and learning. Cell. 2008:135:549-60.

65. Kim S, Kim H, Yim YS, Ha S, Atarashi K, Tan TG, et al. Maternal gut bacteria promote neurodevelopmental abnormalities in mouse offspring. Nature. 2017;549:528-32.

66. Sandquist I, Kolls J. Update on regulation and effector functions of Th17 cells. F1000Res. 2018;7:205.

67. Tausend W, Downing C, Tyring S. Systematic review of interleukin-12, interleukin17, and interleukin-23 pathway inhibitors for the treatment of moderate-tosevere chronic plaque psoriasis: ustekinumab, briakinumab, tildrakizumab, guselkumab, secukinumab, ixekizumab, and brodalumab. J Cutan Med Surg. 2014;18:156-69. 